\title{
Efeitos do exercício físico durante a hemodiálise em indivíduos com insuficiência renal crônica: uma revisão
}

\author{
Effects of physical exercise during hemodialysis in patients with chronic \\ renal insufficiency: a literature review
}

Regina Márcia Faria de Moura ${ }^{1}$, Fernanda Camila Ribeiro Silva ${ }^{2}$, Gláucia Marise Ribeiro ${ }^{2}$, Lidiane Aparecida de Sousa ${ }^{3}$

1 Fisioterapeuta; doutoranda em Ciências da Reabilitação na UFMG (Universidade Federal de Mina Gerais); Profa. do Curso de Fisioterapia do Centro Universitário Newton Paiva, Belo Horizonte, MG

2 Fisioterapeutas

3 Fisioterapeuta; doutoranda em Infectologia e Medicina Tropical na UFMG; Profa. Ms. do Curso de Fisioterapia do Centro Universitário Newton Paiva

ENDEREÇO PARA

CORRESPONDÊNCIA

Regina Márcia Faria de Moura R. Nascimento Gurgel 21 ap.1503 Gutierrez 30430-340 Belo Horizonte MG e-mail:

regimarcia@yahoo.com.br
Resumo: As principais alterações observadas em indivíduos com insuficiência renal crônica são anemia, hipertensão arterial sistêmica e atrofia muscular, que levam à baixa capacidade aeróbica e perda de força muscular. Assim, parte do tratamento desses indivíduos consiste em programas de exercício físico. O objetivo desta revisão da literatura foi documentar os efeitos agudos e as adaptações crônicas, cardiovasculares e musculares em indivíduos no estágio final da doença renal, submetidos a programas de exercício físico durante a hemodiálise. Foram selecionados artigos científicos nas bases eletrônicas Medline, Lilacs e PEDro, assim como no acervo de periódicos da biblioteca da Faculdade de Medicina da UFMG. Foram analisados 13 artigos envolvendo exercício físico aeróbico associado ou não a fortalecimento muscular durante a hemodiálise, variando quanto à intensidade, freqüência e duração da intervenção. A maioria demonstrou que exercícios físicos realizados durante a hemodiálise promovem efeitos benéficos na melhora da capacidade aeróbica, força muscular e no controle dos fatores de risco cardiovasculares, auxiliando a remoção dos solutos durante a hemodiálise. Embora o tema seja ainda pouco explorado, a literatura disponível evidencia benefícios do exercício durante a hemodiálise sobre a capacidade aeróbica e força muscular dos pacientes.

DESCRITORES: Diálise renal; Insuficiência renal crônica; Terapia por exercício

AвSTRACT: Main alterations seen in patients with chronic renal insufficiency are anemia, systemic arterial hypertension, and muscular atrophy, which lead to low aerobic capacity and loss of muscle strength. Hence part of these patients treatment consists in programs of physical exercise. The purpose of this literature review was to assess muscle and cardiovascular acute effects and chronic adaptations in end-stage renal disease patients submitted to physical exercise during hemodialysis. After browsing through Medline, Lilacs and PEDro databases, as well as searching for periodic articles at the UFMG Medicine School library, 13 articles were selected, related to aerobic exercise associated or not to muscle strengthening during hemodialysis. Studies varied as to exercise intensity, frequency and duration. Most articles found that physical exercise performed during hemodialysis favoured better aerobic capacity, muscle strength, and control of cardiovascular risk factors, helping solute removal during hemodialysis. Though the subject is scarcely studied, available literature shows that exercises during hemodialysis are beneficial to patients' aerobic capacity and muscle strength.

Key words: Exercise therapy; Renal dialysis; Renal insufficiency, chronic 


\section{INTRODUÇÃO}

A insuficiência renal crônica (IRC) é considerada um dos principais problemas de saúde no mundo, sendo importante causa de morbidade e mortalidade, caracterizada por perdas lentas, progressivas e irreversíveis das funções renais ${ }^{1-6}$. Hipertensão arterial sistêmica (HAS), diabetes melito e história familiar de doença renal crônica são os fatores de risco mais prevalentes para desenvolvimento da IRC 3-5,7-10,11. Recentes dados do Ministério da Saúde mostram que no Brasil a HAS e o diabetes correspondem a $26 \%$ e $18 \%$ das causas de IRC, respectivamente ${ }^{11}$.

A prevalência da IRC aumenta com a idade e aproximadamente $17 \%$ dos indivíduos com idade acima de 60 anos apresentam maior probabilidade de desenvolver a doença ${ }^{3}$. Sua incidência aumenta cerca de $8 \%$ ao ano no Brasil e a prevalência de indivíduos mantidos em diálise aumentou mais de $100 \%$ nos últimos 8 anos $^{7,4,12}$. Atualmente, há aproximadamente 390 indivíduos/milhão de habitantes em tratamento dialítico ${ }^{12}$.

Pacientes com IRC apresentam menor capacidade física e funcional quando comparados à população geral ${ }^{18}$. Acredita-se que anormalidades musculares e cardiovasculares, comuns nesses indivíduos, contribuam para a ocorrência dessas alterações ${ }^{10,19}$. As anormalidades estruturais e funcionais dos músculos são caracterizadas por miopatia urêmica, que se manifesta como atrofia e fraqueza muscular 10,13,19,20. As causas da miopatia não são bem conhecidas. As possíveis causas descritas na literatura são anemia, miopatia por desuso, alterações no metabolismo energético incluindo metabolismo alterado de carboidratos, diminuição da utilização de lipídios associada à deficiência de carnitina, decréscimo no fluxo sanguíneo muscular, neuropatia periférica e toxinas urêmicas ${ }^{10,19,22}$.

Indivíduos com IRC apresentam risco aumentado para desenvolvimento de doenças cardiovasculares ${ }^{17,21,23,24}$. De acordo com Knap et al. ${ }^{21}$, esse risco é devido principalmente à alta preva- lência de fatores como diabetes melito, dislipidemias, sedentarismo e HAS, sendo que esta última acompanha a IRC em $60 \%$ a $90 \%$ dos $\operatorname{casos}^{8}$.

Em virtude dessas alterações, têm sido propostos programas de exercício físico que visam não somente o tratamento dos sinais clínicos da doença, mas de suas repercussões na função e na qualidade de vida (QV), já que esses indivíduos tendem ao sedentarismo e à limitação funcional ${ }^{14-16}$.

Há aproximadamente 30 anos vem sendo discutida na literatura a utilização de programas de exercícios físicos visando reabilitação física e funcional de indivíduos submetidos à hemodiálise $(\mathrm{HD})^{3}$. Os benefícios citados na literatura incluem melhora da capacidade funcional, redução dos fatores de risco cardiovasculares, melhora da tolerância ao exercício, melhora da tolerância à glicose e de problemas psicossociais 1,14,21,25,26. Apesar de a literatura evidenciar tais resultados, a aplicação de programas de exercícios durante HD na prática clínica ainda é restrita ${ }^{10,13,15,16,27}$.

O objetivo deste estudo foi realizar uma revisão da literatura sobre os efeitos agudos e adaptações crônicas, cardiovasculares e musculares, de indivíduos com IRC submetidos a programas de exercício físico durante HD.

\section{METODOLOGIA}

Foi realizada consulta às bases de dados Medline, Lilacs, PEDro e busca ativa no acervo de periódicos da Biblioteca da Faculdade de Medicina da UFMG em dezembro de 2006, utilizando os seguintes critérios prévios: data de publicação entre janeiro de 1996 e dezembro de 2006; idiomas, português e inglês; unitermos incluídos no título e/ou resumo - hemodiálise (hemodialysis), estágio final da doença renal (end-stage renal disease), exercício (exercise) e força muscular (muscle strength); estudos experimentais relacionados à IRC, $\mathrm{HD}$ e exercício físico durante a HD.

Para a seleção dos artigos, inicial- mente foi feita a leitura dos resumos, verificando se continham as informações que preenchiam os seguintes critérios de inclusão: estudos com participantes adultos (maiores de 18 anos) em tratamento de HD por mais de três meses, em uso ou não de eritropoetina (EPO); estudos que avaliaram os efeitos de programas de exercícios constituídos de treinamento aeróbico e/ou fortalecimento muscular durante HD. Os estudos que, além de participantes em tratamento hemodialítico, também envolviam participantes em tratamento de diálise peritoneal, foram incluídos, porém somente os resultados dos participantes em HD foram considerados. Os artigos da seleção final foram incluídos e lidos na íntegra.

\section{RESULTADOS}

Foram analisados 13 artigos, dos quais $11^{18,23,29,30-37}$ avaliaram as adaptações crônicas ao exercício e dois estudos avaliaram os efeitos agudos ${ }^{17,38}$. Apenas um estudo envolveu grupo de participantes em HD e em diálise peritoneal, porém os resultados foram analisados separadamente ${ }^{18}$.

A HAS, diabetes melito e glomerulonefrite foram as principais causas de IRC citadas nos estudos ${ }^{17,18,23,29,30-38}$. A duração total de intervenção variou de seis semanas a quatro anos, sendo o treinamento aeróbico a modalidade de exercício mais utilizada. Os principais dados referentes aos estudos selecionados estão sintetizados no Quadro 1.

\section{DISCUSSÃO}

Os estudos selecionados são aqui comentados segundo se refiram aos efeitos agudos e adaptações crônicas de programa de exercícios físicos realizados durante a HD.

\section{Efeitos agudos do exercício físico}

Kong et al. ${ }^{38}$, em ensaio clínico aleatorizado, demonstraram que 60 minutos de exercício aeróbico intermitente durante a HD promoveram aumento significativo da remoção de 


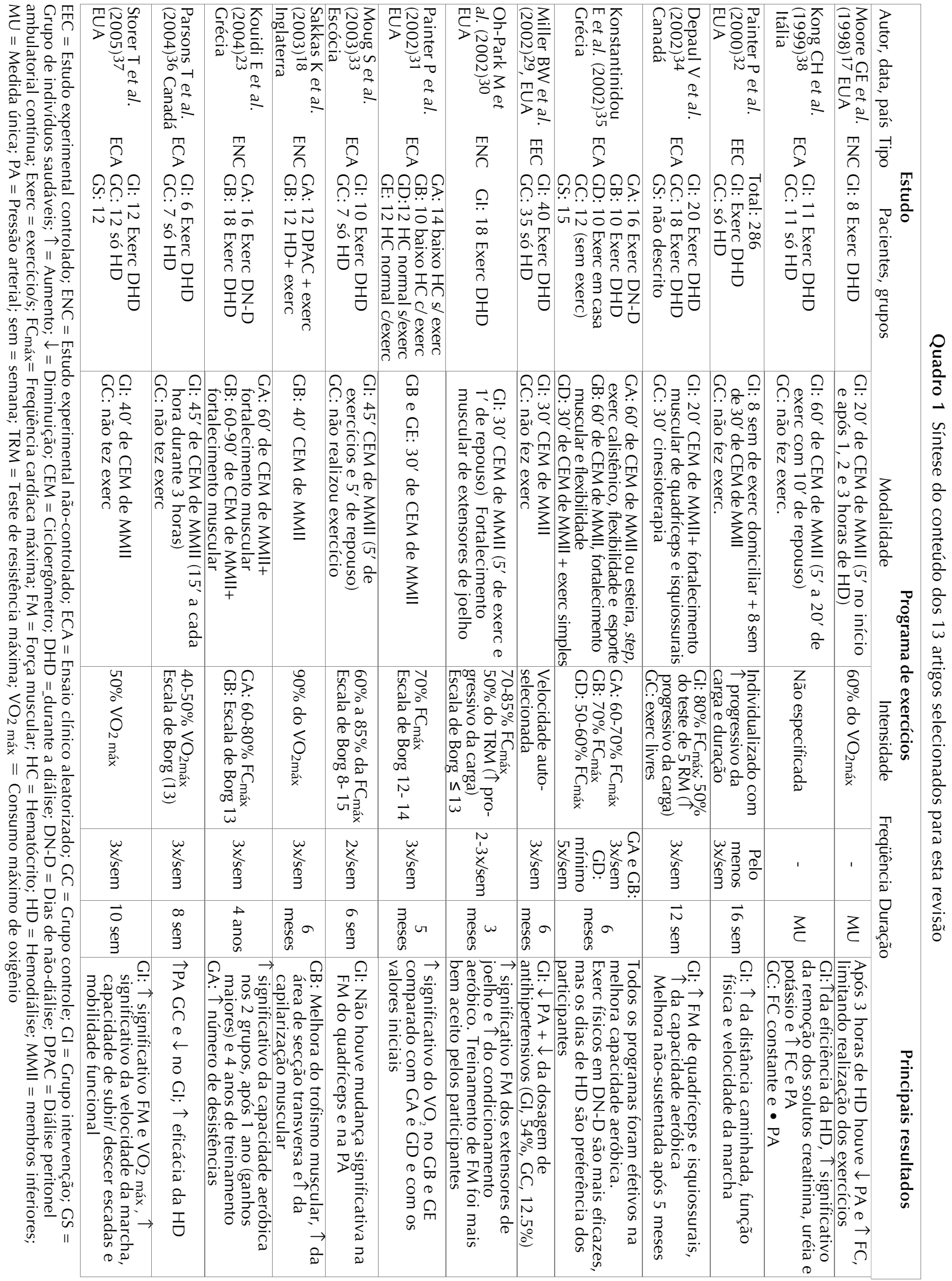


três solutos - uréia, creatinina e potássio - no grupo intervenção, melhorando a eficiência da HD e redução de sua duração em 30 minutos. O possível mecanismo do aumento significativo da remoção dos solutos é a vasodilatação que ocorre na musculatura esquelética, propiciando aumento na remoção de catabólitos. Os autores salientam que mais pesquisas são necessárias para confirmar tais achados.

Moore et al. ${ }^{17}$ descreveram as respostas cardiovasculares ocorridas durante o exercício aeróbico intermitente realizado no início, após uma, duas e três horas de HD, totalizando 20 minutos de exercício. Após três horas de HD, as alterações cardiovasculares ocorridas com o exercício foram hipotensão e taquicardia, limitando a continuação do exercício. Uma possível causa da hipotensão, segundo os autores, é que indivíduos com IRC apresentam maior ativação do sistema nervoso simpático associado a uma diminuição dos receptores alfa-adrenérgicos, favorecendo ocorrência da hipotensão durante a HD. Outra possível explicação para a hipotensão é que muitas vezes a remoção do soluto durante a HD ultrapassa a capacidade do organismo desses indivíduos urêmicos de compensar a perda excessiva de volume, por apresentarem alteração do sistema nervoso simpático. Segundo os autores, esses mecanismos são pouco conhecidos, sendo necessários mais estudos para elucidá-los.

Em decorrência dos efeitos deletérios induzidos pelo exercício realizado após três horas de HD, a maioria dos estudos subseqüentes conduziu os programas de exercícios nas primeiras duas e até três horas de $\mathrm{HD}^{30,33-38}$.

Os efeitos agudos adversos decorrentes do exercício físico durante HD citados nos estudos foram: dispnéia, fadiga, dor nas pernas, câimbras e hipoglicemia. Os autores não mencionaram em qual momento da sessão essas alterações ocorreram, nem suas possíveis causas ${ }^{17,23,29,30,32,36}$. Portanto, a realização de atividades físicas duran- te a HD deve incluir monitoramento rigoroso de parâmetros clínicos e hemodinâmicos dos pacientes.

\section{Adaptações crônicas}

Embora tenham ocorrido grandes avanços em relação ao tratamento da IRC, o tratamento dialítico isolado não assegura melhora física e funcional desses indivíduos. Portanto, a incorporação de exercícios físicos a sua rotina deve ser considerada ${ }^{1}$.

Sakkas et al. ${ }^{18}$ investigaram os efeitos de seis meses de exercícios aeróbicos durante a HD na morfologia do músculo gastrocnêmio de indivíduos com IRC. Foram evidenciados após treinamento aumento da área de secção transversa (46\%) e redução da atrofia das fibras musculares tipo I (51\% para $15 \%)$, tipo IIA (58\% para $21 \%$ ) e tipo IIB (62\% para $32 \%$ ). Diferenças significativas também foram encontradas com relação ao aumento da capilarização muscular.

Storer et al. ${ }^{37}$ avaliaram os efeitos do treinamento aeróbico com intensidade de $50 \%$ do consumo máximo de oxigênio, na força muscular e na performance física. Os autores demonstraram que houve melhora da força bem como do desempenho (performance) físico no grupo intervenção, mesmo com baixa intensidade de exercício aeróbico, realizado com cicloergômetro de membros inferiores. Esses resultados podem ser explicados pela marcada fraqueza muscular e pelo baixo condicionamento cardiorrespiratório apresentado por esses indivíduos. Apesar da melhora apresentada pelos participantes que realizavam os exercícios, o consumo máximo de oxigênio permaneceu 30\% abaixo daquele do grupo de indivíduos saudáveis. Os autores afirmam que é possível que o exercício físico em curto prazo, com baixa intensidade, em indivíduos com marcada miopatia urêmica e outras comorbidades, não seja suficiente para restabelecer função aeróbica igual à de indivíduos saudáveis.

Depaul et al. ${ }^{34}$ verificaram melhora da força muscular e capacidade aeróbica no grupo intervenção após 12 semanas de tratamento. Os resultados dos testes de força muscular e de caminhada de 6 minutos aproximaram-se dos valores de indivíduos saudáveis, porém essa melhora não foi sustentada 5 meses após o tratamento. Oh-Park et al. ${ }^{30}$, em estudo envolvendo treinamento aeróbico associado ao fortalecimento muscular, observaram melhora significativa da força muscular dos extensores de joelho e da capacidade aeróbica. Nesse estudo, o fortalecimento muscular foi mais bem aceito pelos participantes do que o treinamento aeróbico.

Estudos têm documentado efeitos de programas de exercícios físicos no controle dos fatores de risco cardiovasculares $^{28,31,34,36}$ e diminuição do uso de medicamentos anti-hipertensivos ${ }^{29}$. Miller et al. ${ }^{29}$, em estudo controlado, verificaram que exercícios aeróbicos durante a HD promoveram redução do uso de anti-hipertensivos de 54\% no grupo intervenção e de 12,5\% no grupo controle, após 6 meses de treinamento. Corroborando esses resultados, Parsons et al. ${ }^{36}$ demonstraram benefícios nas adaptações do sistema cardiovascular no grupo de participantes em tratamento dialítico que realizou exercícios aeróbicos, comparado ao grupo controle. Os autores relatam significativa redução da pressão arterial no grupo intervenção, porém não mencionaram redução da dosagem dos anti-hipertensivos.

A avaliação da QV vem sendo incorporada aos desfechos de estudos envolvendo pacientes com IRC ${ }^{1}$. Painter et al. ${ }^{32}$, em estudo controlado de 16 semanas de exercício aeróbico individualizado, demonstraram que houve aumento da distância caminhada e velocidade da marcha, bem como melhora da função física avaliada pelo questionário Medical Outcomes Study Short-form 36 (SF-36) no grupo intervenção, comparado ao grupo controle, que não realizou exercícios. Oh-Park et al. ${ }^{30}$ também evidenciaram melhora dos escores do componente mental do SF-36 após treinamento aeróbico durante HD. 
Segundo a Fundação Nacional do Rim norte-americana, que publicou diretrizes para o tratamento da anemia na doença renal crônica (2000, apud Depaul et al. ${ }^{34}$ ), a eritropoetina (EPO) tem sido amplamente utilizada em indivíduos submetidos à HD, com objetivo de manter os níveis de hemoglobina entre 11 e 12 g/dl. Estudos de Depaul et al. ${ }^{34}$ e Painter et al. ${ }^{31}$ documentaram que o uso de EPO combinado ao exercício promoveu melhora da tolerância ao exercício, aumento do consumo de oxigênio e redução da fadiga.

Konstantinidou et al. ${ }^{34}$ compararam os efeitos de três modelos de treinamento: em dias de não-HD, durante a HD, e domiciliar. A capacidade aeróbica melhorou significativamente nos três modelos de treinamento. Os resultados foram mais evidentes no treinamento em dias de não-HD quando comparados ao de dias de HD e domiciliar. Nesse estudo a taxa de desistência no grupo que realizava os exercícios nos dias de não HD foi de aproximada- mente $24 \%$, em razão das dificuldades de transporte e falta de tempo, enquanto no grupo que realizava os exercícios durante a HD foi de $17 \%$.

Kouidi et al. ${ }^{23}$ compararam dois programas de exercícios: o grupo A fazia exercícios nos dias de não-HD e o grupo B, durante a HD. Os resultados mostraram que houve melhora significativa da capacidade aeróbica e da QV após 1 ano e após 4 anos de intervenção, nos dois grupos. O número de desistências foi maior no grupo $\mathrm{A}$ $(37,5 \%)$ comparado ao grupo B (21\%). Exercícios realizados durante a HD mostraram ser de preferência pelos mesmos motivos descritos por Konstantinidou et al. ${ }^{34}$. Com base nos resultados desses dois estudos ${ }^{28,34}$, pode-se inferir que as duas formas de intervenção promovem benefícios, porém as desistências e dificuldades encontradas pelos indivíduos para fazer exercício físico nos dias em que não vão à hemodiálise são maiores.

Em apenas um estudo, de Moug et al. ${ }^{33}$, verificou-se que, após seis semanas de intervenção durante a HD, não houve melhora estatisticamente significativa da capacidade aeróbica, nem da força muscular do quadríceps. Os autores defendem que o treinamento aeróbico promove benefícios para os indivíduos com IRC, mas acreditam que o curto período de intervenção foi o responsável pelos achados.

\section{CONCLUSÃO}

Após extensa revisão da literatura pode-se concluir que o exercício físico realizado durante a hemodiálise promove benefícios físicos e funcionais. Há diversidade quanto à forma de aplicação desses programas em termos de intensidade, freqüência e duração, devendo estas ser adequadas às realidades de cada serviço e de cada paciente. Por se tratar de um tema ainda pouco explorado, mais estudos são necessários para confirmação desses achados.

\section{REFERENCIAS}

1 Cheema BSB, Singh MAF. Exercise training in patients receiving maintenance hemodialysis: a systematic review of clinical trials. Am J Nephrol. 2005;25(4):352-64.

2 Hsu C, Vittinghoff E, Lin F, Shlipak MG. The incidence of end-stage renal disease is increasing faster than the prevalence of chronic renal insufficiency. Ann Intern Med. 2004;141(2):95-101.

3 Johnson CA, Levey AS, Coresh J, Levin A, Lau J, Eknoyan G. Clinical practice guidelines for chronic kidney disease in adults, part I: definition, disease stages, evaluation, treatment, and risk factors. Am Family Physician. 2004;70(6):869-75.

4 Romão Junior JE. Doença renal crônica: definição, epidemiologia e classificação. J Bras Nefrol. 2004;26:1-3.

5 Rahman M, Smith MC. Chronic renal insufficiency: a diagnostic and therapeutic approach. Arch Intern Med. 1998;158(16):1743-52.
6 Viktorsdottir O, Palsson R, Andresdottir MB, Aspelund $\mathrm{T}$, Gudnason V, Indridason OS. Prevalence of chronic kidney disease based on estimated glomerular filtration rate and proteinuria in Icelandic adults. Nephrol Dial Transplant. 2005;20(9):1799-1807.

7 Bastos MG, Carmo WB, Abrita RR, Almeida EC, Mafra D, Costa DMN, et al. Doença renal crônica: problemas e soluções. J Bras Nefrol. 2004;26(4):202-15.

8 Beltrame RE, Guilhen JC, Araújo IM, Zanolli MB. Perfil dos pacientes com IRC: análise retrospectiva de 221 casos no Hospital das Clínicas da Faculdade de Medicina de Marília. Rev Bras Med. 2000;57:1-12.

9 Bregman R. Prevenção da progressão da doença renal crônica. J Bras Nefrol. 2004;26:11-3.

10 Deligiannis A. Exercise rehabilitation and skeletal muscle benefits in hemodialysis patients. Clin Nephrol. 2004;61(Suppl 1):S46-50. 


\section{Referências (cont.)}

11 Romão Jr JE, Pinto SWL, Canziani ME, Praxedes JN, Santello JL, Moreira JCM. Censo SBN 2002: informações epidemiológicas das unidades de diálise do Brasil. J Bras Nefrol. 2003;25(4):188-9.

12 Oliveira MB, Romão Jr JE, Zatz R. End-stage renal disease in Brazil: epidemiology, prevention, and treatment. Kidney Int. 2005;68:1-5.

13 Johansen KL, Shubert T, Doyle J, Soher B, Sakkas GK, Braun A. Muscle atrophy in patients receiving hemodialysis: effects on muscle strength, muscle quality, and physical function. Kidney Int. 2002;63:291-7.

14 Daul AE, Schafers RF, Daul K, Philipp T. Exercise during hemodialysis. Clin Nephrol. 2004;61(Suppl 1):S26-30.

15 Duarte PS, Mitazaki MCOS, Ciconelli RM, Sesso R. Tradução e adaptação cultural do instrumento de avaliação de qualidade de vida para pacientes renais crônicos (KDQOL-SF). Rev Assoc Med Bras. 2003;49(4):375-81.

16 Martins MRI, Cesarino CB. Atualização sobre programas de educação e reabilitação para pacientes renais crônicos submetidos à hemodiálise. J Bras Nefrol. 2004;26(1):45-50.

17 Moore GE, Painter PL, Brinker KR, Stray-Gundersen J, Mitchell JH. Cardiovascular response to submaximal stationary cycling during hemodialysis. Am J Kidney Dis. 1998;31(4):631-7.

18 Sakkas GK, Sargeant AJ, Mercer TH, Ball D, Koufaki P, Karatzaferi $\mathrm{C}$, et al. Changes in muscle morphology in dialysis patients after 6 months of aerobic exercise training. Nephrol Dial Transplant. 2003;18(9):1854-61.

19 Moreira PR, Barros E. Atualização em Fisiologia e Fisiopatologia Renal: bases fisiopatológicas da miopatia na insuficiência renal crônica. J Bras Nefrol. 2000;22(1):40-4.

20 Painter P. Physical functioning in end-stage renal disease patients: update 2005. Hemodial Int. 2005;9(3):218-35.

21 Knap B, Ponikvar JB, Ponikvar P, Bren AF. Regular exercise as a part of treatment for patients with endstage renal disease. Ther Apher Dial. 2005;9(3):211-3.

22 Fahal I, Bell GM, Bone JM, Edwards RHT. Physiological abnormalities of skeletal muscle in dialysis patients. Nephrol Dial Transplant. 1997;12(1):119-27.

23 Kouidi E, Grekas D, Deligiannis A, Tourkantonis A. Outcomes of long-term exercise training in dialysis patients: comparison of two training programs. Clin Nephrol. 2004;61(Suppl 1):S31-8.

24 Deligiannis A. Cardiac adaptations following exercise training in hemodialysis patients. Clin Nephrol. 2004;61(Suppl 1):S35-45.
25 Moore G. Prescrição de exercício na insuficiência renal. In: Shankar K. Prescrição de exercícios. Rio de Janeiro: Guanabara Koogan; 2000. Cap. 7, p.105-9.

26 Oberley ET, Sadler JH, Alt PS. Renal rehabilitation: obstacle, progress, and prospects for the future. Am J Kidney Dis. 2000;35(4 Suppl1):S141-7.

27 Medeiros RH, Pinent CEC, Meyer F. Aptidão física de indivíduo com doença renal crônica. J Bras Nefrol. 2002;24(2):81-7.

28 Kouidi E. Exercise training in dialysis patients: why, when, and how? Artif Organs. 2002;26(12):1009-13.

29 Miller BW, Cress CL, Johnson ME, Nichols DH, Schnitzler MA. Exercise during hemodialysis decreases the use of antihypertensive medications. Am J Kidney Dis. 2002;39(4):828-33.

30 Oh-Park M, Fast A, Gopal S, Lynn R, Frei G, Drenth $R$, et al. Exercise for the dialyzed: aerobic and strength training during hemodialysis. Am J Phys Med Rehabil. 2002;81(11):814-21.

31 Painter P, Moore G, Carlson L, Paul S, Myll J, Phillips W, et al. Effects of exercise training plus normalization of hematocrit on exercise capacity and health-related quality of life. Am J Kidney Dis. 2002;39(2):257-65.

32 Painter P, Carlson L, Carey S, Paul S, Myll J. Physical functioning and health-related Quality-of-life changes with exercise training in hemodialysis patients. Am J Kidney Dis. 2000;35(3):482-92.

33 Moug SJ, Grant S, Creed G, Boulton JM. Exercise during hemodialysis: West of Scotland pilot study. Scott Med J. 2003;49(1):14-7.

34 DePaul V, Moreland J, Eager T, Clase CM. The effectiveness of aerobic and muscle strength training in patients receiving hemodialysis and EPO: a randomized controlled trial. Am J Kidney Dis. 2002;40(6):1219-29.

35 Konstantinidou E, Koukouvou G, Kouidi E, Deligiannis A, Tourkantonis A. Exercise training in patients with end-stage renal disease: comparison of three exercise programs. J Rehabil Med. 2002;34(1):40-5.

36 Parsons TL, Toffelmire EB, King-VanVlack CE. The effect of an exercise program during hemodialysis on dialysis efficacy, blood pressure and quality of life in end-stage renal disease (ESRD) patients. Clin Nephrol. 2004;61(4):261-74.

37 Storer TW, Casaburi R, Sawelson S, Kopple JD. Endurance exercise training during haemodialysis improves strength, power, fatigability and physical performance in maintenance hemodialysis patients. Nephrol Dial Transplant. 2005;20(7):1429-37.

38 Kong CH, Tattersall JE, Greenwood RN, Farringyon G. The effect of exercise during haemodialysis on solute removal. Nephrol Dial Transplant. 1999;14(12):2927-31. 